Original Contribution

\title{
OPEN REDUCTION AND FIXATION OF FRACTURES OF THE HEAD AND NECK OF THE HUMERUS IN CHILDREN WITH THE USE OF INTRAMEDULLARY ELASTIC OSTEOSYNTHESIS
}

\author{
N. Dimitrov ${ }^{1 *}$, T. Al-Sadek ${ }^{2}$, A. Dimitrov ${ }^{1}$, E. Simeonov ${ }^{4}$ \\ ${ }^{1}$ Department of Orthopaedics and Traumatology, University Hospital "Prof. Dr. Stoyan Kirkovich", \\ Stara Zagora, Bulgaria \\ ${ }^{2}$ Department of Orthopaedics and Traumatology, Belhoul European Hospital, Dubai, UAE \\ ${ }^{4}$ Department of Orthopaedics and Traumatology, University Hospital "G.Stranski", Pleven, Bulgaria
}

\begin{abstract}
Radial neck fractures are not common (5-10\%) but are very difficult to treat and have many possible complications. [1, 2] In the conservative treatment it's impossible to reduce severe displaced fractures, resulting in malfunction. Open reduction frequently leads to intra articular calcification, avascular necrosis, and joint stiffness. [3] In most cases, intramedullary nailing allows complete reduction without capsulotomy and very low complication rate of infection.[4] The pin is left in the bone, stabilizes the epiphysis, and prevents secondary displacement. [8, 9]
\end{abstract}

Keywords: fracture, radial neck, radial head, children, pinning.

\section{INTRODUCTION}

Fractures in the proximal part of the radius are epiphyseal lesions of the head and neck metaphysical fractures. They occupy about 5$10 \%$ of all traumatic lesions in the elbow joint in children. $(1,2,3)$ These fractures are difficult to treat and have many possible complications that affect the function of the elbow joint. Removal of the radial head is now completely abandoned a method of treatment in childhood. Surgical reposition indicated in some cases of severe displacements. Surgical method of treatment, however, is associated with complications, so that's why the first choice for treatment in the conservative treatment. Despite the large number of reposition methods proposed lately, reposition it is not always possible and the risk of secondary displacement of the fracture is too high.

The elastic stable intramedullary osteosynthesis (ESIN) was introduced in 1980 by French authors. $(4,5,6)$ It consists in introducing long and slightly curved at the top titanium pin in the intramedullary radial canal percutaneously distal opening up to the

\footnotetext{
* Correspondence to: Nedko Dimitrov, $M D, P h D$, Department of Orthopedics and Traumatology, Medical Faculty, Trakia University, Stara Zagora, Bulgaria,e-mail:dimitrovnedko@yahoo.com
}

proximal part. Reaching the lower aspect of the epiphysis of the proximal radius, the titanium needle elevates the fragment up, with the rotation of the needle axis fragment repositions and the needle remains in this position for a period of 6 to 8 months. The main advantage of this method is that it allows both precise and stable reposition and stable fixation of the fragments retaining periosteal vascularity of the radial head.

\section{Anatomy of the proximal radius}

The blood supply of the radial head is through the periosteum. The whole epiphysis is surrounded by the articular cartilage so that the vessels can only enter into the bone through the periosteum. The supplying one or two arteries have a short intra articular entrance along the metaphysis, which is covered by the annular ligament. Fractures of the neck of the radius raptures a part of the periosteum vessels and reduce the blood supply to the head. Surgical approaches lead to an increased risk of damage to the periosteal vessels, leading to necrosis of the radial head.

\section{Clinical Case}

14 years old girl, with trauma in the right elbow joint from a fall, with abducted hand There is a fracture of the radial neck, third degree classification of Judet (7), with displaced fragments kept intact and angulation 
between the fragments of $40^{\circ}$. In this clinical case valgus stress has led to additional avulsion lesion from the medial side of the

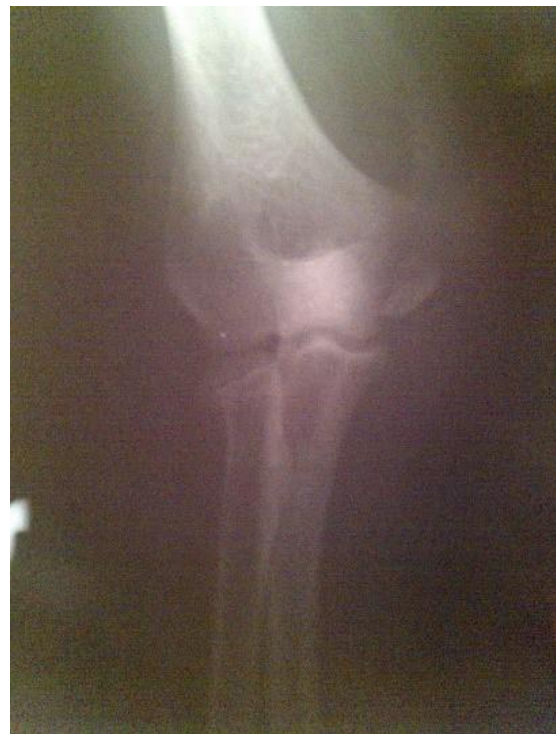

joint - apophysiolysis of the ulnar epicondyle.

(Figure 1A, B)

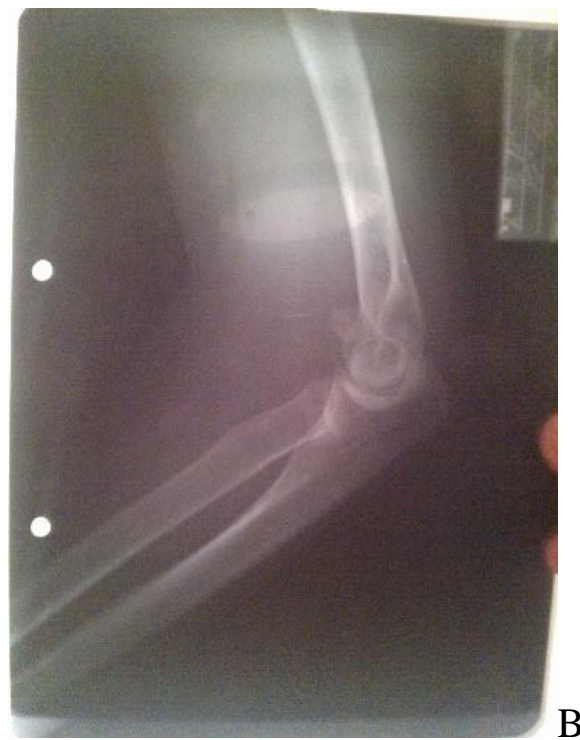

Figure 1A, B. Diagnostic X-ray of the right elbow AP view and lateral view

\section{Surgical technique}

Closed reduction of the radial neck with elevation and rotation of the fragment and holding the fracture intramedullary with a Kirschner wire $2.5 \mathrm{~mm}$, distaly under X-ray control.The Ulnar epicondyle is then repositioned by an open reduction and fixation with a K -wire and an AO - screw. Plaster immbilization is not needed post operatively.

Surgical approach for intramedullary fixation of the radial neck is $1 \mathrm{~cm}$ skin incision passing along the lateral border of the distal radial metaphysis $1 \mathrm{~cm}$ proximal to the epiphysial plate. The soft tissues are disinserted with scissors and a periosteal elevaor avoiding superficial veins and in particular the superficial branch of the Radial nerve. Periosteum and the insertion of brachioradial $\mathrm{m}$. bare in the space of a few $\mathrm{mm} 2$. With bone awl going into the cortex $2 \mathrm{~cm}$ proximal to distal physis and directs proximal to the long axis of the radius. The preliminary prepared TEN (titanium elastic nail) is introduced into the medullary canal of the radius. With the tap is directed upwards so that its tip to move to the lower aspect of the fracture. At this stage with gentle hammer blows to TEN-tip and fixed in the epiphysis, elevates it till it settles into the lateral condyle of the humerus, which acts as a buffer to prevent recorrection. When the fracture is adjusted, the nail is rotated around its long axis so that its peak be rotated and further adjustment of the proximal fragment. All this is monitored under X-ray control. The lower metaphyseal end to TEN is cut and the skin is closed (Figure 2).

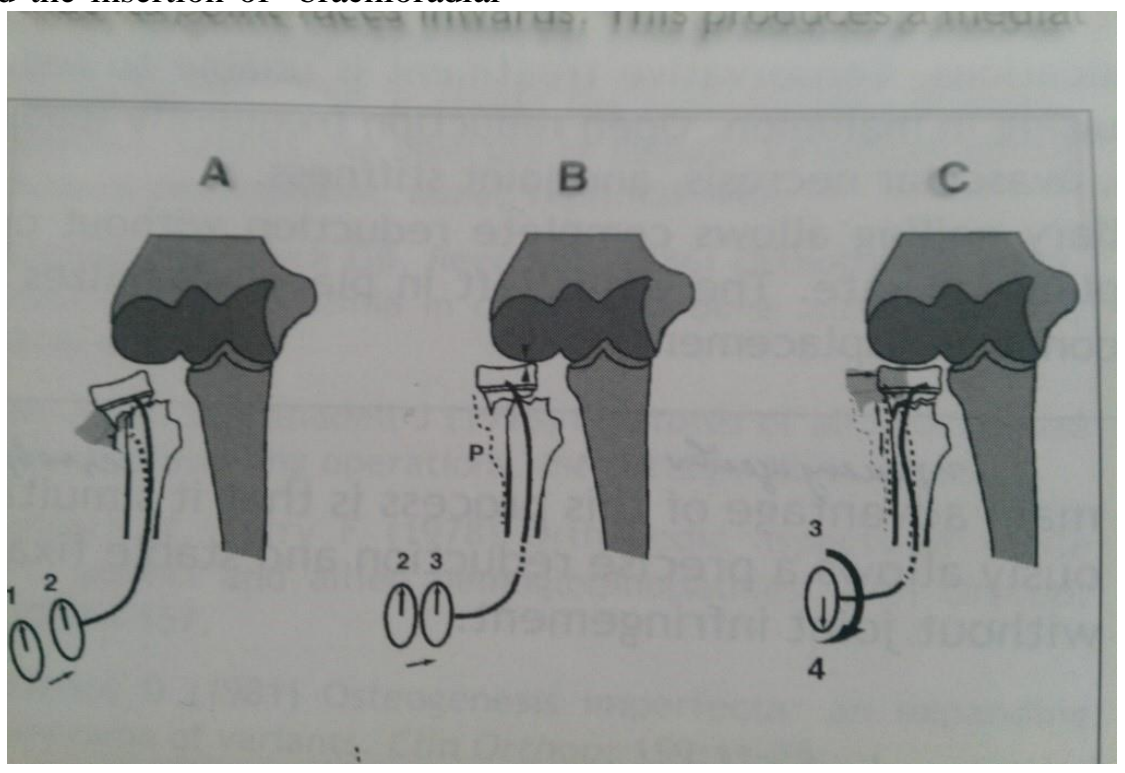

Figure 2. Schematic representation of the sequence of intramedullary osteosynthesis of the radial neck. 
Postoperative period in this clinical case proceeded smoothly, without complications and without the use of additional plaster immobilization. Stitches from the surgical

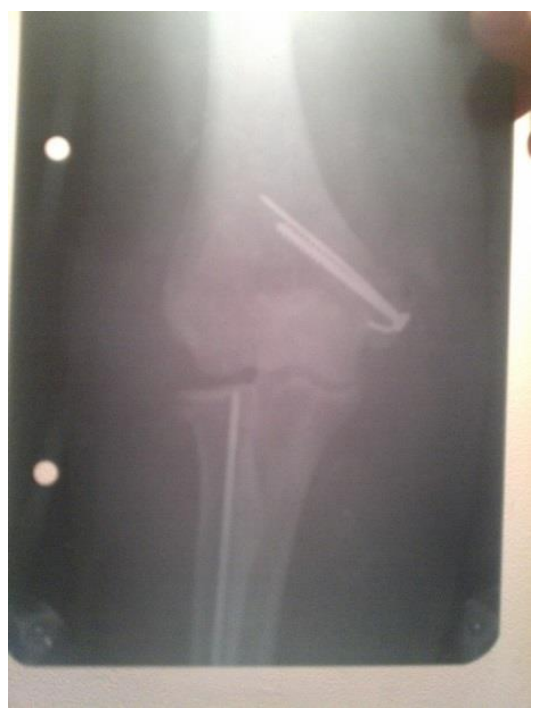

A wound were removed on the 12th postoperative day. Arm sling is applied for sparing and immobilization in the first three weeks after surgery (Figures 3A, B and 4).

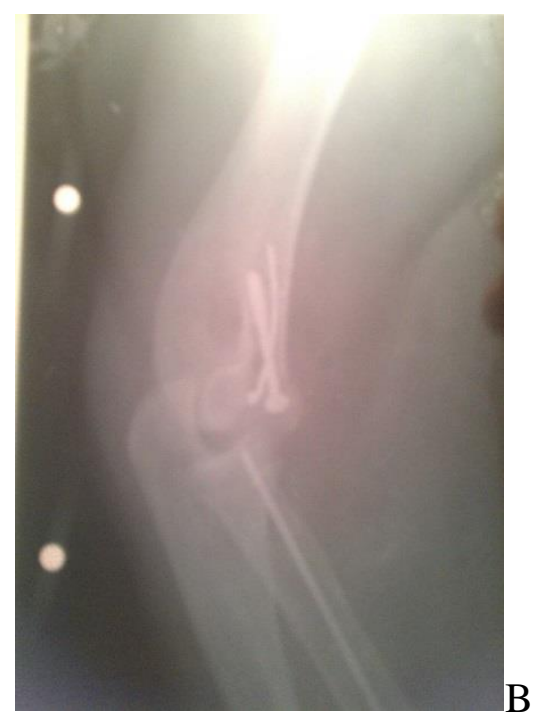

Figure 3A, B. Postoperative X-ray immediately after surgery in AP and lateral view.

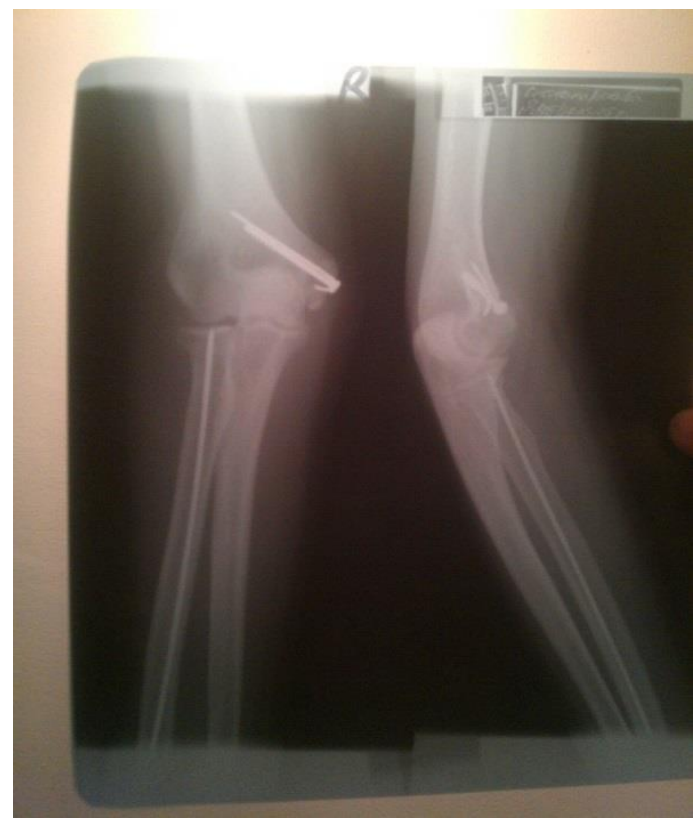

Figure 4. Post-operative X-ray 8 months after surgery in AP and lateral view.

\section{CONCLUSIONS}

Intramedullary osteosynthesis with TEN for treatment of fractures of the radial head and neck is a minimally invasive technique that leads to good and stable reposition and avoid most of the complications: malunion, avascular necrosis, calcification (which rarely occurs after conservative treatment), progressive kubitus valgus, painful movements.[8;9] Children's fractures of the head and neck radius require special attention and minimally invasive approach to preserve periosteal blood supply to the radial head. (10) Fractures of the ulnar epicondyle heal without complications after anatomical reposition.

\section{REFERENCES}

1. Angelov A. A., Traumatic injuries (sprains and isolated fractures and dislocations) of the proximal end of the radius in children. Thesis. Sofia, 1977.

2. A. Mihailov et al., Absolute indications for surgical treatment of fresh fractures of the neck of the radius in children. Orthotrauma 30:11, 1993.

3. Novkov H; Takov E., Fractures in Children 324: 375, 2000.

4. Gonzalez-Herranz P., Alvarez-Romera A., Burgos J., et al., Displaced radial neck fractures in children treated by closed 
intramedullary pinning. J Pediatr Orthop; 17 (3): 325-331, 1997.

5. Henrikson B., Isolated fractures of the proximal end of the radius. Epidemiology, treatment and prognosis. Acta Orthop Scand; 40 (2): 246-260, 1969.

6. Jones E.R.L., Esam M., Displaced fractures of the neck of the radius in children. $J$ Bone Joint Surg; 52-A: 314-324, 1971.

7. Judet J., Judet R., Lefranc J., Fractures of the radial head in the child. Ann Chir; 16: 1377-1385, 1962.

8. Metaizeau J.P., Prevot J., Schmitt M., Reduction and ficsation of fractures of the neck of the radius by centromedullary pinning. Original Technique. Rev Chir Orthop Reparatrice Appar Mot; 66 (1): 47 49, 1980.

9. Metaizeau J.P., Prevot J., Finlayson D., et al. Reduction and fixation of displaced radial neck fractures by closed intramedullary pinning. $J$ Pediatr Orthop; 13 (3): 355-360, 1993.

10.Reidy J.M., Vangorder G.W. , Treatment of displacement of the proximal radial epiphysis. J Bone Joint Surg; 45-A: 13551372, 1963. 